\title{
El nivel educativo como factor asociado al bienestar subjetivo y la salud mental en la población española*
}

\author{
Educational Level as a Member of the Subjective Well- \\ Being and Mental Health in the Spanish Population
}

Recibido: noviembre 6 de 2011 | Revisado: febrero 2 de 2012 | Aceptado: marzo 19 de 2012

\author{
ERnesto De la CRUZ-SÁNCHEZ** \\ Universidad de Murcia, España \\ SEBASTIÁN FEU **** \\ MANUEL VizUETE-CARRIZOSA **** \\ Universidad de Extremadura, España
}

\section{RESUMEN}

El objetivo de este trabajo fue evaluar la asociación entre el nivel educativo alcanzado con el bienestar emocional y la salud mental de los españoles. Un total de 29.478 personas adultas participaron en la Encuesta Nacional de Salud de España 2006, encontrándose que un nivel educativo bajo se asocia con peor salud mental (OR 1.65 hombres y OR 2.36 mujeres), valoración mala o muy mala de la salud (OR 2.10 hombres y OR 2.53 mujeres), menor apoyo social (OR 1.31 hombres y OR 1.66 mujeres) y sedentarismo (OR 2.09 hombres y OR 1.80 mujeres). En la muestra estudiada, el nivel educativo es un factor asociado a diferentes dimensiones de la salud relacionadas con el bienestar subjetivo y la salud mental.

\section{Palabras clave autores}

Condiciones sociales, calidad de vida, apoyo social, actividad física, estilo de vida,

España.

Palabras clave descriptores

Psicología de la salud, Encuesta Nacional de Salud de España.

Para citar este artículo: De la Cruz-Sánchez, E., Feu, S. \& Vizuete-Carrizosa, M. (2013). El nivel educativo como factor asociado al bienestar subjetivo y la salud mental en la población española. Universitas Psychologica, 12(1), 31-40.

* Agradecimientos: Los autores agradecen al Ministerio de Sanidad y Política Social - Instituto de Información Sanitaria de España la disponibilidad de los datos de la Encuesta Nacional de Salud de España. Los análisis y el contenido del presente trabajo son responsabilidad única de los autores firmantes.

** Campus de San Javier. E-mail: erneslacruz@um.es

**** Facultad de Educación. E-mail: sfeu@unex.es

${ }^{* * * * *}$ Facultad de Educación. E-mail: mvizuete@unex.es

\begin{abstract}
A B S T R A C T
The aim of this study was to evaluate the association between the educational level with the perceived emotional well-being and the mental health of the Spanish population. In a total of 29,478 adults, participating in the Spanish National Health Survey 2006, we found that low education level is related with poor mental health (OR 1.65 in men and OR 2.36 in women), poor or very poor self-perceived health (OR 2.10 OR 2.53 for men and women), less social support (OR 1.31 OR 1.66 for men and women) and inactivity (OR 2.09 OR 1.80 for men and women). In the Spanish population the educational level is an associated factor related with different health dimensions and to the subjective well-being and mental health perceptions. Key words authors

Social conditions, quality of life, social support, physical activity, lifestyle, Spain. Key words plus

Health Psychology, National Survey of Health of Spain.
\end{abstract}




\section{Introducción}

La promoción de la salud contempla dimensiones no ligadas a enfermedad. Según la Organización mundial de la Salud ([OMS], 1946), "La salud es un estado completo de bienestar físico y mental, y no solamente la ausencia de enfermedades". Este enfoque global de la salud debería tener una consideración mayor de la que actualmente se le otorga, ya que problemas relacionados con la salud mental y el bienestar emocional de la población suponen creciente gasto sanitario, y este tipo de problemas serán, a corto y medio plazo, la principal causa de discapacidad y de absentismo laboral (Roberts \& Grimes, 2011). Esto significa que la estimación de la salud de la población exige evaluar, además, factores asociados a la autopercepción del bienestar psicológico y emocional, y de la integración social. Aspectos, que en su mayor parte, vienen determinados por el contexto socioeconómico en el que la persona se desenvuelve (Laaksonen et al., 2007).

Desde una perspectiva de salud pública, existe una serie de determinantes sociales que condicionan la salud, y desde hace 40 años se viene describiendo la existencia de desigualdades socioeconómicas relacionadas con la morbilidad y la mortalidad (Marmot, Rose, Shipley \& Hamilton, 1978).

El estatus social de la persona se suele definir esencialmente en términos laborales y económicos, atributos estos que, en su mayor parte, están asociados al nivel educativo alcanzado. La importancia del nivel educativo como indicador de desigualdades sociales en salud, en ocasiones, ha sido subestimado o ni siquiera considerado en muchos trabajos, siendo a nuestro modo de ver un factor que condiciona y justifica, en gran medida, la existencia de estas desigualdades y que sería preciso tener en cuenta en este tipo de estudios (d'Uva, O'Donnell $\&$ van Doorslaer, 2008).

El empleo del nivel educativo como indicador del estatus socioeconómico tiene, además, algunas ventajas sobre estos tradicionales indicadores económicos de clase. La educación de una persona es un indicador de estatus estable a lo largo de la vida, y permite clasificar a personas que, o bien no trabajan o están jubiladas, lo que descarta la idea de que el estatus social pueda resultar condicionado por el estado de salud de una persona y por la exposición a factores de estrés o problemas de salud, tanto más probables a medida que aumenta la edad (Huisman et al., 2005).

Existen evidencias que señalan que el nivel educativo podría ser el factor socioeconómico que mejor describa las desigualdades sociales en salud (Regidor, Gutiérrez-Fisac, Domínguez, Calle \& Navarro, 2002), y ya ha sido descrita la asociación entre este indicador y diferentes causas específicas de mortalidad (Regidor, Calle, Navarro \& Domínguez, 2003) y morbilidad hospitalaria (Bautista, Alfonso, Corella \& Saiz, 2005).

La relación entre la salud percibida y la mortalidad puede, por tanto, resultar condicionada por el nivel educativo. Las personas que tienen un nivel educativo bajo tienden a valorar peor su salud, al verse afectadas en mayor medida que otras por problemas crónicos y, por tanto, en ellas la mortalidad no está tan asociada con esta valoración propia de la salud; mientras que en las personas con un nivel educativo alto la salud percibida es un indicador mejor relacionado al del riesgo de mortalidad (Regidor, Guallar-Castillón, Gutiérrez-Fisac, Banegas \& Rodríguez-Artalejo, 2010).

Independientemente de la clase social basada en la ocupación laboral y del estatus socioeconómico relacionado con el nivel de ingresos, la mejora del sistema educativo tiende a disminuir las desigualdades y a equiparar a toda la población en términos de salud, y puede contrarrestar el factor de riesgo que supone tener, en edades tempranas, un estatus socioeconómico bajo o crecer en un hogar con bajos ingresos (McKenzie, Carter, Blakely \& Ivory, 2011).

De la misma forma, es necesario señalar que actualmente un nivel educativo bajo es un signo de exclusión social cada vez más importante, ya que las diferencias en salud en función del nivel educativo que hemos descrito en este estudio son cada vez mayores en las generaciones más recientes (Regidor et al., 2002).

Así, la educación debería ser uno de los factores clave para cualquier política orientada al desarrollo del bienestar de los ciudadanos: un buen sistema educativo, accesible en sus distintos niveles a toda la 
población y especialmente a los colectivos más desfavorecidos, constituye uno de los pilares del estado del bienestar en cuanto que puede hacer disminuir las desigualdades sociales estructurales en salud, afectando en forma positiva y directa a las conductas y toma de decisiones que fundamentan el estilo de vida ciudadano y permitiendo el empoderamiento y socialización de la población, de forma que esta sea más consciente de sus problemas y obtenga más recursos para resolverlos (Navarro, 2009).

El objetivo del presente trabajo desde una perspectiva integral de la salud fue explorar la relación existente entre el nivel de educación formal alcanzado y diferentes indicadores de bienestar autorreferidos: percepción subjetiva de salud, salud mental, aceptación y soporte social y actividad física durante el tiempo libre.

\section{Método}

\section{Diseño del estudio y participantes}

Los datos empleados proceden de la Encuesta Nacional de Salud de España (ENSE- 2006), un estudio transversal desarrollado por el Ministerio de Sanidad y Política Social de España en el que se evalúa la salud de un total de 29.478 personas (11.645 hombres y 17.833 mujeres mayores de 16 años), representativas del total de la población española. La muestra se dividió en 50 submuestras provinciales, empleando un muestreo polietápico y estratificado por conglomerados en el que se tuvo en cuenta en primer lugar, las secciones censales; en segundo, los hogares y finalmente un individuo de cada hogar. Las secciones censales fueron elegidas dentro de cada estrato con probabilidad proporcional a su tamaño, mientras que los hogares e individuos fueron seleccionados de manera aleatoria teniendo en cuenta cuotas de edad y sexo (establecidas a partir del último censo oficial disponible).

Para minimizar sesgos estacionales en términos de morbilidad y estilo de vida, la entrevista se condujo en cuatro etapas de 15 días, y el período de referencia de cada variable explorada estuvo compuesto por 2 semanas y 1 año, desde el día de la recogida de datos. La recolección de información se llevó a cabo por entrevistadores previamente formados para la tarea.

\section{Variables}

El nivel educativo se obtuvo preguntando a cada entrevistado acerca de su educación formal completada, recodificándose posteriormente en tres niveles a partir de los criterios UNESCO-ISCED, en: bajo (sin estudios o estudios primarios), medio (estudios secundarios y formación profesional de grado medio) y alto (formación profesional de grado superior, primer ciclo universitario y segundo ciclo universitario), UNESCO (1976). En la Figura 1 se encuentra representado el porcentaje de población que ha completado cada nivel educativo en función de su edad, tanto de hombres como mujeres (nótese que en la franja de edad que va de los 16 a los 24 años existe un alto porcentaje de personas que por su edad aún no han podido finalizar sus estudios, por encontrarse escolarizadas).

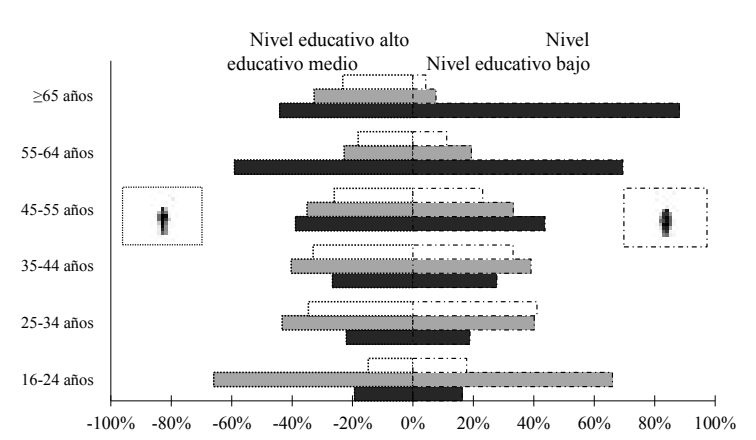

Figura 1. Distribución por edad y sexo del nivel educativo en la población objeto de estudio, según la clasificación UNESCO-ISCED.

Fuente: elaboración propia a partir de los datos de la ENSE 2006.

La ENSE 2006 contiene una escala de valoración de la propia salud percibida que ha sido recategorizada en tres factores (mala o muy mala, regular y buena o muy buena), y además incluye un índice de salud mental subjetivo obtenido mediante un cuestionario que consta de 12 preguntas (General Health Questionnaire [GHQ-12]), diseñado originalmente para el diagnóstico de trastornos 
psiquiátricos (Goldberg et al., 1997), ampliamente utilizado en la bibliografía internacional y validado para la población española (Sánchez-López \& Dresch, 2008). La puntuación obtenida oscila de 0 a 12 puntos: de mejor salud mental a peor salud mental. Para facilitar la comparación estadística en este estudio las personas son agrupadas en percentiles, conteniendo el percentil 95 a las personas con peor salud mental.

El apoyo social ha sido estimado a través del cuestionario Duke-UNC-11 en su versión española (Bellón, Delgado, Luna \& Lardelli, 1996). Este cuestionario es una herramienta que permite cuantificar el soporte social de una persona en dos dimensiones del mismo: confidencial (personas con las que se comparten sentimientos íntimos) y emocional (personas que expresan sentimientos de empatía con nosotros). El punto de corte para diagnosticar bajo apoyo social ha sido establecido en el percentil 15, que en nuestra muestra se corresponde con puntuaciones inferiores a 41 .

\section{TABLA 1}

Descriptivos básicos en función del sexo de los diferentes indicadores de bienestar estudiados ( $\mathrm{N}$ porcentaje)

\begin{tabular}{lcc}
\hline & Hombres & Mujeres \\
\hline Salud Mental & & \\
Percentil 50 & $9.363(84.3)$ & $12.438(72.6)$ \\
Percentil 75 & $1.450(13.1)$ & $3.888(22.7)$ \\
Percentil 95 & $297(2.7)$ & $798(4.7)$ \\
Salud Percibida & & \\
Buena o muy buena & $7.764(69.9)$ & $9.840(57.5)$ \\
Regular & $2.476(22.3)$ & $5.221(30.5)$ \\
Mala o muy mala & $870(7.8)$ & $2.063(12.0)$ \\
Apoyo Social (DUKE-UNC 11) & \\
Alto & $4.754(42.8)$ & $7.646(44.7)$ \\
Medio & $4.338(39.0)$ & $6.574(38.4)$ \\
Bajo & $1.735(15.6)$ & $2.549(14.9)$ \\
Perdidos & $283(2.5)$ & $355(2.1)$ \\
Actividad Física durante el tiempo libre & \\
Practica actividad & $7.081(63.7)$ & $9.920(57.9)$ \\
física & $3.988(35.9)$ & $7.147(41.7)$ \\
Sedentarismo & $41(0.4)$ & $57(0.3)$ \\
\hline
\end{tabular}

Fuente: elaboración propia a partir de los datos de la ENSE 2006.
Finalmente, la práctica de actividad física en el tiempo libre fue obtenida por medio de este cuestionario mediante la siguiente pregunta: "Habitualmente, irealiza en su tiempo libre alguna actividad física como caminar, hacer algún deporte, gimnasia...?" Esta pregunta podía ser contestada por el entrevistado de forma dicotómica "sí" o "no, normalmente no hago ejercicio físico". En la Tabla 1 se puede observar la distribución y prevalencia de las diferentes variables analizadas en la muestra estudiada.

\section{Análisis estadístico}

Las estimaciones fueron ponderadas para tener en cuenta el diseño muestral. Se presentan porcentajes con la distribución de los indicadores de salud favorables en función del nivel educativo completado, y se calculó además la tasa de instrucción incompleta para cada cohorte de edad, dividiendo el número de personas con bajo nivel educativo entre el número total de personas (se presenta en porcentaje). De la misma forma, en cada cohorte de edad se calculó la fracción atribuible a un nivel educativo bajo en la población para la prevalencia de un percentil 95 de salud mental, mala salud percibida, bajo apoyo social y sedentarismo, como FAP = It-Io/It, siendo It la incidencia del indicador negativo en la población, e Io = Incidencia del indicador negativo en la población con un nivel educativo medio y alto.

Finalmente, se calcularon las odds ratio y sus respectivos intervalos de confianza al $95 \%$ a través de un análisis de regresión logística multinomial para establecer el grado de asociación entre las variables que han sido objeto de estudio, utilizando como variable dependiente el nivel educativo alcanzado y como variables independientes los diferentes indicadores seleccionados. Para el análisis se utilizó el programa SPSS versión 19.0.

\section{Resultados}

Un análisis descriptivo de la prevalencia de buena salud en los indicadores estudiados ya muestra un sesgo favorable hacia las personas con un nivel educativo superior, tal y como puede apreciarse en 

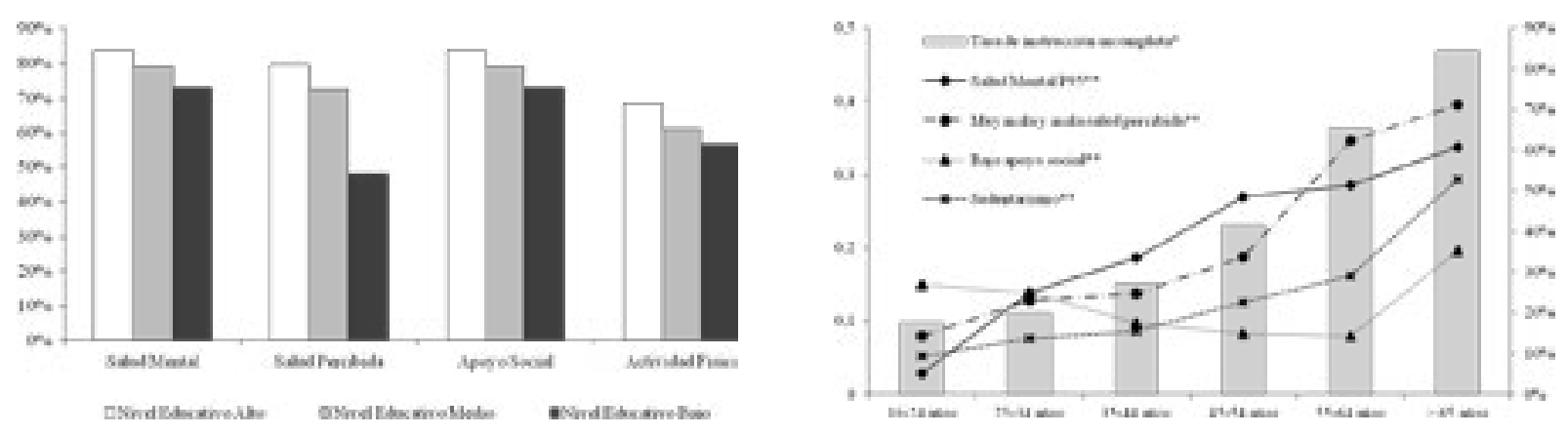

Figura 2. Prevalencia en la población española de un percentil 50 de salud mental, buena o muy buena salud percibida, apoyo social alto y práctica de actividad física durante el tiempo libre en función del nivel educativo alcanzado (\%).

Fuente: elaboración propia a partir de los datos de la ENSE 2006.

la Figura 2. Al comparar los tres niveles se constata la existencia de un marcado gradiente de salud en función del nivel educativo, que en todos los casos es proporcionalmente creciente al nivel de educación formal completada.

El análisis detallado del nivel de instrucción de la población, por cohortes de edad, refleja la historia de la alfabetización en España y la implantación

Figura 3. Representación gráfica de la tasa de instrucción incompleta por cohortes de edad (eje de la derecha) y fracción atribuible en la población (FAP, eje de la izquierda) analizando, por cohortes de edad y en cada variable, la contribución de un nivel educativo bajo a la prevalencia de indicadores negativos de bienestar.

Fuente: elaboración propia a partir de los datos de la ENSE 2006.

progresiva de un modelo educativo más accesible a los ciudadanos, disminuyendo la tasa de instrucción incompleta de la población estudiada un $74 \%$ en los últimos 40 años (barras del histograma de la Figura 3). Las tres generaciones educadas durante la democracia que recoge este trabajo tienen una

\section{TABLA 2}

Resultados del modelo de regresión logística multinomial (OR y 95 \% IC) examinando en españoles adultos la asociación entre el nivel educativo y las variables de bienestar estudiadas

\begin{tabular}{|c|c|c|c|c|}
\hline & \multicolumn{2}{|c|}{ Hombres - Nivel Educativo $^{1}$} & \multicolumn{2}{|c|}{ Mujeres - Nivel Educativo ${ }^{1}$} \\
\hline & Medio & Bajo & Medio & Bajo \\
\hline \multicolumn{5}{|l|}{ Salud Mental ${ }^{2}$} \\
\hline Percentil 75 & $1.27(1.08-1.50) *$ & $1.27(1.07-1.49) *$ & $1.36(1.21-1.52)^{*}$ & $1.41(1.25-1.58) *$ \\
\hline Percentil 95 & $1.80(1.21-2.66)^{*}$ & $1.65(1.13-2.41)^{*}$ & $1.60(1.19-2.15)^{*}$ & $2.36(1.78-3.13) *$ \\
\hline \multicolumn{5}{|l|}{ Salud Percibida ${ }^{3}$} \\
\hline Regular & $1.56(1.34-1.81)^{*}$ & $2.10(1.82-2.42)^{*}$ & $1.57(1.41-1.76)^{*}$ & $2.06(1.84-2.30) *$ \\
\hline Mala o muy mala & $1.56(1.21-2.01)^{*}$ & $2.10(1.67-2.64)^{*}$ & $1.52(1.25-1.85)^{*}$ & $2.53(2.11-3.04) *$ \\
\hline \multicolumn{5}{|c|}{ Apoyo Social (DUKE-UNC 11) } \\
\hline Medio & $0.98(0.87-1.09)$ & $1.08(0.96-1.21)$ & $1.09(0.99-1.20)$ & $1.15(1.04-1.27)^{*}$ \\
\hline Bajo & $1.14(0.97-1.34)$ & $1.31(1.12-1.54)^{*}$ & $1.32(1.14-1.52) *$ & $1.66(1.44-1.92) *$ \\
\hline \multicolumn{5}{|l|}{ Actividad Física ${ }^{5}$} \\
\hline Sedentarismo & $1.45(1.29-1.62) *$ & $2.09(1.87-2.34) *$ & $1.33(1.22-1.46) *$ & $1.80(1.64-1.98) *$ \\
\hline
\end{tabular}

Nota. Odds ratio ajustada a la edad e ingresos económicos del hogar. "Grupo de comparación es "Nivel educativo alto"; ${ }^{1} \mathrm{Gru}$ po de comparación es "Percentil 50 (buena salud mental)"; "Grupo de comparación es "Salud percibida buena o muy buena"; ${ }^{4}$ Grupo de comparación es "Apoyo Social alto"; ${ }^{5}$ Grupo de comparación es "Práctica de actividad física durante el tiempo libre". $* p<0.01$.

Fuente: elaboración propia a partir de los datos de la ENSE. 
tasa de instrucción incompleta un $62 \%$ inferior a la cohorte de población de mayor edad.

Además de observar una importante reducción de la tasa de instrucción incompleta en las generaciones más jóvenes, lo que es un indicador de la mejora social en términos educativos, se observa en la población estudiada que la fracción atribuible al nivel educativo bajo para valores negativos de bienestar y salud mental disminuye de forma similar a esta tasa de instrucción incompleta (líneas de tendencia, Figura 3). Tal es el caso de la FAP para la contribución de un nivel educativo bajo a una mala salud mental (P95), la autovaloración de la salud como mala-muy mala y un estilo de vida sedentario. En el caso del bajo apoyo social, la FAP para la contribución de un nivel educativo bajo disminuye solo hasta la tercera generación de más edad.

El gradiente social en los diferentes indicadores de bienestar estudiados se mantiene cuando analizamos estadísticamente su asociación con el nivel educativo alcanzado (Tabla 2), excepto en el caso del apoyo social, en el que no se observa una relación tan marcada, como es el caso de la población masculina, ya que los hombres que tienen un nivel educativo medio no difieren de los que tienen un nivel educativo alto. No obstante, la asociación estadísticamente significativa entre un nivel educativo bajo y valores negativos en las variables estudiadas se mantiene indistintamente en todo caso (Tabla 2).

En función del sexo, la diferencia entre hombres y mujeres estriba en que, si bien en ambos se observa este gradiente de asociación entre nivel educativo y estos indicadores de bienestar, es en estas en las que un nivel educativo bajo se asocia más con peores resultados en los diferentes indicadores: para el percentil 95 de salud mental la diferencia va de una OR 1.65 en hombres a una OR 2.36 en mujeres; en el caso de la valoración mala o muy mala de la propia salud va de una OR 2.10 a una OR 2.53 y en lo que respecta a la valoración de apoyo social, el grupo con nivel educativo bajo presenta una $\mathrm{OR}$ 1.31 (hombres) y una OR 1.66 (mujeres). La excepción es la actividad física: los hombres con un nivel educativo bajo presentan mayor probabilidad de ser sedentarios (OR 2.09) que las mujeres de este mismo nivel educativo (OR 1.80), quizá debido a que ser mujer es, en sí mismo, un factor asociado a mayor prevalencia de sedentarismo en la muestra estudiada independientemente del nivel educativo (OR 1.26 y $p<0.01$, ajustada para edad y nivel de estudios).

\section{Discusión}

El gradiente social en salud basado en el nivel educativo, que ha sido ampliamente descrito en relación con el uso de servicios médicos y la prevalencia de enfermedades no transmisibles (Wilkinson \& Marmot, 2003), también es observado en estos aspectos psicológicos, emocionales y conductuales ligados al bienestar. Un trabajo similar que ha evaluado la calidad de vida en nuestro país en función del nivel educativo apunta conclusiones parecidas (Regidor et al., 1999), y en este sentido el presente estudio viene a complementar la descripción de esta relación al valorar dimensiones hasta ahora menos exploradas como son la salud mental o el apoyo social percibido. No obstante, en el estudio citado algunas dimensiones de la calidad de vida de los españoles (estimada a través del inventario SF-36) no muestran este gradiente de asociación tan marcado y existen diferencias sustanciales en función del sexo (Regidor et al., 1999), mientras que en el presente trabajo, independientemente de la edad o sexo, la salud mental y el bienestar subjetivo muestran una asociación positiva con el nivel educativo alcanzado.

Más concretamente relacionado con el objetivo del presente estudio, el declive cognitivo, la depresión y la incidencia de patologías psiquiátricas como la demencia senil o Alzheimer son más frecuentes en personas que han tenido menos años de escolarización (Chazelle et al., 2011; Reuser, Willekens $\&$ Bonneux, 2011). Los factores psicosociales, la maestría y la autoeficacia relacionadas con el nivel educativo alcanzado explican la mayor parte de las diferencias educativas en el funcionamiento físico y mental a lo largo de la vida, no siendo tan determinantes la carestía material o el acceso a bienes de consumo (Groffen et al., 2012). Como hemos señalado en este estudio, en la población española se da esta asociación entre nivel educativo y salud 
mental, independientemente de otro tipo de indicadores de estatus socioeconómico como puede ser el nivel familiar de ingresos.

Gran parte de los trabajos que exploran las diferencias socioeconómicas en salud suelen incluir la valoración de la propia salud en sus estimaciones y, al igual que en este estudio, se observa la relación entre mayor nivel educativo y mejor salud percibida a lo largo de toda la vida (Foraker et al., 2001). La respuesta a una simple pregunta acerca del propio estatus de salud proporciona una información básica pero que se relaciona estrechamente con aspectos tan relevantes como la mortalidad, morbilidad, calidad de vida y conductas relacionadas con la salud (Singh-Manoux et al., 2007).

De igual forma, en la población estudiada las personas con un nivel educativo alto perciben mayor apoyo social, lo que en términos de salud se traduce en mayor bienestar y calidad de vida: el apoyo social es un factor relacionado estrechamente con el estado general de salud (Melchior, Berkman, Niedhammer, Chea \& Goldberg, 2003) y tiene una incidencia directa sobre la salud mental y la calidad de vida de las personas (De la Revilla, de los Ríos Álvarez \& de Dios Luna del Castillo, 2007; Stansfeld, 2006). Esta asociación positiva entre el soporte social y nivel educativo que describimos se mantiene en la mayoría de países europeos, tanto en hombres como en mujeres, aunque la magnitud de esta asociación varíe en función de la edad, de cada país y del sexo (Von dem Knesebeck \& Geyer, 2007).

La inclusión en el presente estudio de la práctica de actividad física durante el tiempo libre como variable a analizar obedece a que se puede emplear la prevalencia de la misma como un indicador de bienestar: la práctica de actividad física se relaciona con la funcionalidad física de una persona (Stewart et al., 1994) e incluso con su salud mental, ya que un estilo de vida activo es menos frecuente en personas con trastornos emocionales y mala salud mental (De la Cruz-Sánchez, Moreno-Contreras, Pino-Ortega \& Martínez-Santos, 2011; RodríguezHernández, De la Cruz-Sánchez, Feu \& MartínezSantos, 2011). Los resultados del presente trabajo son coherentes con un estudio previo en el que se observa que las personas con un nivel educativo bajo o menor nivel de ingresos tienen peor condición física relacionada con la salud (Saavedra et al., 2008). El gradiente social en el mantenimiento de un estilo de vida activo ya ha sido descrito, resaltando que las diferencias en la práctica de actividad física en función del nivel educativo son más determinantes que las diferencias debidas a la clase social en función de la ocupación profesional o el estatus laboral (Mäkinen et al., 2010).

Uno de los principales sesgos que se dan en el presente trabajo es que al ser un estudio transversal no se puede inferir estadísticamente el efecto del nivel educativo sobre la salud mental y el bienestar subjetivo a lo largo de la vida de una persona. En la muestra estudiada, el efecto de la edad es más que plausible en la prevalencia de mala salud mental, peor valoración de la propia salud, pérdida de apoyo social y declarar un estilo de vida sedentario; no obstante, podemos observar cómo a pesar de que la tasa de instrucción incompleta disminuye en cada generación a medida que disminuye la edad (Figura 3), el riesgo de presentar peores resultados para un nivel educativo bajo se mantiene en el total de la población independientemente de la edad (Tabla 2). Estos resultados apuntan a que el nivel educativo de la población podría ser uno de los elementos clave de la mejora de la calidad de vida en las últimas décadas, y que las generaciones más jóvenes de ciudadanos se benefician del incremento generalizado del nivel educativo cada vez mayor.

El presente estudio, realizado en una amplia base poblacional y con el objetivo de definir el gradiente social en términos de salud mental y bienestar subjetivo, es uno de los pocos entre los países de Iberoamérica que tratan esta temática concreta. Los resultados constatan la existencia de un marcado gradiente social en diferentes dimensiones de salud no sanitarias, y ponen de relieve que no solo existen desigualdades sociales en términos de esperanza de vida, morbilidad y mortalidad, sino que también el estatus educativo de una persona es un factor potencialmente determinante, en gran medida, de su salud mental y bienestar. El gradiente social, en términos educativos, es un problema potencialmente más abordable desde una perspectiva de salud 
pública que otros factores, como son los ingresos familiares o la ocupación laboral, y la existencia de un sistema educativo público de calidad puede contribuir a mejorar la salud de los ciudadanos, en su más amplia acepción.

\section{Referencias}

Bautista, D., Alfonso, J. L., Corella, D. \& Saiz, C. (2005). Influence of social factors on avoidable mortality: A hospital-based case-control study. Public Health Reports, 120(1), 55-62.

Bellón, J. A., Delgado, A., Luna, J. D. \& Lardelli, P. (1996). Validez y fiabilidad del cuestionario de apoyo social funcional Duke-UNC-11. Atención Primaria, 18(4), 153-163.

Chazelle, E., Lemogne, C., Morgan, K., Kelleher, C. C., Chastang, J. F. \& Niedhammer, I. (2011). Explanations of educational differences in major depression and generalized anxiety disorder in the Irish population. Journal of Affective Disorders, 134(1), 304-314.

De la Cruz-Sánchez, E., Moreno-Contreras, M. I., PinoOrtega, J. \& Martínez-Santos, R. (2011). Leisure time physical activity and its relationships with some mental health indicators in Spain through the National Health Survey. Salud Mental, 34(1), 45-52.

De la Revilla, L., de los Ríos Álvarez, A. M. \& de Dios Luna del Castillo, J. (2007). Factors underlying psycho-social problems. Atención Primaria, 39(6), 305-311.

d'Uva, T. B., O'Donnell, O. \& van Doorslaer, E. (2008). Differential health reporting by education level and its impact on the measurement of health inequalities among older Europeans. International Journal of Epidemiology, 37(6), 1375-1383. doi:10.1093/ije/dyn146

Foraker, R. E., Rose, K. M., Chang, P. P., McNeill, A. M., Suchindran, C. M., Selvin, E., et al. (2011). Socioeconomic status and the trajectory of self-rated health. Age and Ageing, 40(6), 706-711. doi:10.1093/ ageing/afr069

Goldberg, D. P., Gater, R., Sartorius, N., Ustun, T. B., Piccinelli, M., Gureje, O., et al. (1997). The validity of two versions of the GHQ in the WHO study of mental illness in general health care. Psychological Medicine, 27(1), 191-197.

Groffen, D. A., Bosma, H., Tan, F. E., van den Akker, M., Kempen, G. I. \& van Eijk, J. T. (2012). Material vs. psychosocial explanations of old-age educational differences in physical and mental functioning. European Journal of Public Health, 22(1), 587-592. doi: 10.1093/eurpub/ckr063

Huisman, M., Kunst, A. E., Bopp, M., Borgan, J. K., Borrell, C., Costa, G., et al. (2005). Educational inequalities in cause-specific mortality in middleaged and older men and women in eight western European populations. Lancet, 365(9458), 493-500. Laaksonen, E., Martikainen, P., Lahelma, E., Lallukka, T., Rahkonen, O., Head, J., et al. (2007). Socioeconomic circumstances and common mental disorders among Finnish and British public sector employees: Evidence from the Helsinki Health Study and the Whitehall II Study. International Journal of Epidemiology, 36(3), 776-786. doi:10.1093/ ije/dym074

Mäkinen, T. E., Sippola, R., Borodulin, K., Rahkonen, O., Kunst, A., Klumbiene, J., et al. (2010). Explaining educational differences in leisure-time physical activity in Europe: The contribution of work-related factors. Scandinavian Journal of Medicine \& Science in Sports, 22(3), 439-447. doi: 10.1111/j.1600. 0838.2010.01234.x

Marmot, M. G., Rose, G., Shipley, M. \& Hamilton, P. J. (1978). Employment grade and coronary heart disease in British civil servants. Journal of Epidemiology and Community Health, 32(4), 244-249.

McKenzie, S. K., Carter, K. N., Blakely, T. \& Ivory, V. (2011). Effects of childhood socioeconomic position on subjective health and health behaviours in adulthood: How much is mediated by adult socioeconomic position? BMC Public Health, 11, 1-10. doi:10.1186/1471-2458-11-269

Melchior, M., Berkman, L. F., Niedhammer, I., Chea, M. $\&$ Goldberg, M. (2003). Social relations and self-reported health: A prospective analysis of the French Gazel cohort. Social Science and Medicine, 56(8), 1817-1830. doi:10.1016/S0277-9536(02)00181-8

Navarro, V. (2009). What we mean by social determinants of health. International Journal of Health Ser- 
vices: Planning, Administration, Evaluation, 39(3), 423-441.

Organización Mundial de la Salud. (1946). Constitución de la Organización Mundial de la Salud. New York. Recuperado de http://www.who.int/governance/eb/ constitution/es/index.html

Regidor, E., Barrio, G., de la Fuente, L., Domingo, A., Rodríguez, C. \& Alonso, J. (1999). Association between educational level and health related quality of life in Spanish adults. Journal Epidemiology and Community Health, 53(2), 75-82.

Regidor, E., Calle, M. E., Navarro, P. \& Domínguez, V. (2003). The size of educational differences in mortality from specific causes of death in men and women. European Journal of Epidemiology, 18(5), 395-400.

Regidor, E., Guallar-Castillón, P., Gutiérrez-Fisac, J. L., Banegas, J. R. \& Rodríguez-Artalejo, F. (2010). Socioeconomic variation in the magnitude of the association between self-rated health and mortality. Annals of Epidemiology, 20(5), 395-400. doi:10.1016/j.annepidem.2010.01.007

Regidor, E., Gutiérrez-Fisac, J. L., Domínguez, V., Calle, M. E. \& Navarro, P. (2002). Comparing social inequalities in health in Spain: 1987 and 1995/97. Social Science Medicine, 54(9), 1323-1332.

Reuser, M., Willekens, F. J. \& Bonneux, L. (2011). Higher education delays and shortens cognitive impairment: A multistate life table analysis of the US Health and Retirement Study. European Journal of Epidemiology, 26(5), 395-403. doi: 10.1007/ s10654-011-9553-x

Roberts, G. \& Grimes, K. (2011). Return on investment mental health promotion and mental illness prevention. Ottawa: Canadian Institute for Health Information.

Rodríguez-Hernández, A., De la Cruz-Sánchez, E., Feu, S. \& Martínez-Santos, R. (2011). Sedentarismo, obesidad y salud mental en la población española de 4 a 15 años de edad. Revista Española de Salud Pública, 85(4), 373-382.

Saavedra, J. M., Torres, S., Caro, B., Escalante, Y., De la Cruz, E., Durán, M. J., et al. (2008). Relationship between health-related fitness and educational and income levels in Spanish women. Public Health, 122(8), 794-800. doi:10.1016/j.puhe.2007.07.017

Sánchez-López, M. D. \& Dresch, V. (2008). The 12-Item General Health Questionnaire (GHQ-12): Reliability, external validity and factor structure in the Spanish population. Psicothema, 20(4), 839-843.

Singh-Manoux, A., Guéguen, A., Martikainen, P., Ferrie, J., Marmot, M. \& Shipley, M. (2007). Selfrated health and mortality: Short- and long-term associations in the Whitehall II study. Psychosomatic Medicine, 69(2), 138-143. doi:10.1097/ PSY.0b013e318030483a

Stansfeld, S. A. (2006). Social support and social cohesion. In M. Marmot \& R. G. Wilkinson (Eds.), Social determinants of health (pp. 148-171). Oxford: Oxford University Press.

Stewart, A. L., Hays, R. D., Wells, K. B., Rogers, W. H., Spritzer, K. L. \& Greenfield, S. (1994). Long-term functioning and well-being outcomes associated with physical activity and exercise in patients with chronic conditions in the Medical Outcomes Study. Journal of Clinical Epidemiology, 47(7), 719 730. doi:10.1016/0895-4356(94)90169-4

United Nations Educational Scientific and Cultural Organization. (1976). International Standard Classification of Education (ISCED). París: Autor.

Von dem Knesebeck, O. \& Geyer, S. (2007). Emotional support, education and self-rated health in 22 European countries. BMC Public Health, 7, 272. doi:10.1186/1471-2458-7-272

Wilkinson, R. \& Marmot, M. (2003). Social determinants of health: The solid facts. Copenhague: World Health Organization. 
